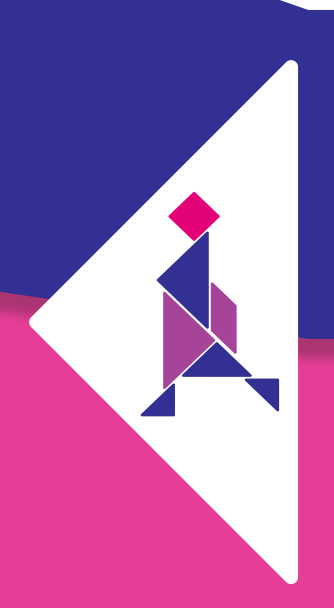

\title{
Efeitos de um programa de fisioterapia na qualidade de vida, no equilíbrio, na flexibilidade e na força de preensão de idosas
}

Effects of a program physycal therapy

on quality of life balance, flexibility

and elderly grip strength

Fisisenectus . Unochapecó Ano 3, n. 1 - Jan/Jun. 2015 p. $3-10$

Tainá Pesente. tainapsnt@hotmal.com

Acadêmica do $10^{\circ}$ semestre do Curso de Fisioterapia da Universidade Regional Integrada do Alto Uruguai e das Missões - Erechim.

Fabrizzio Pelle. fabrizziopelle@yahoo.com.br

Fisioterapeuta. Docente da Universidade Regional Integrada do Alto Uruguai e das Missões - Erechim.

Zequiela Russi. zequirussi@yahoo.com.br

Fisioterapeuta. Docente da Universidade Regional Integrada do Alto Uruguai e das Missões - Erechim.

Caren Maronezzi. carentpiccoli@hotmail.com

Fisioterapeuta. Docente da Universidade Regional Integrada do Alto Uruguai e das Missões - Erechim.

Janesca Mansur Guedes. janescaguedes@yahoo.com.br

Fisioterapeuta. Docente da Universidade Regional Integrada do Alto Uruguai e das Missões - Erechim.

\section{Resumo}

Nos últimos tempos, observa-se um aumento da população idosa, diante disso e cientes do aumento das doenças crônico-degenerativas e também dos malefícios do sedentarismo, estratégias são necessárias para manter a população idosa ativa. Atividades físicas supervisionadas por fisioterapeutas visam à preservação das funções, promovendo um modo de vida mais saudável e, consequentemente, o aumento da qualidade de vida. 0 objetivo deste estudo foi verificar os efeitos de um programa de fisioterapia na qualidade de vida, no equilíbrio, na flexibilidade e na força de preensão manual em um grupo de idosas. Como avaliação, inicialmente, foi aplicado o questionário Whoqol-brev. Após o teste do banco de wells, foi utilizado o teste up and go e para finalizar o dinamômetro manual. Os participantes foram avaliados no início e após nove meses do programa de exercícios fisioterapêuticos, durante 25 sessões, sendo que os testes foram realizados em grupo, uma vez por semana, com duração de cinquenta minutos. Os resultados demonstram melhora estatisticamente significativa da percepção do estado de saúde, da flexibilidade, do equilíbrio e da força de preensão manual de MSD. Conclui-se que um programa de exercícios fisioterapêuticos de longo prazo é um meio eficaz para a manutenção da qualidade de vida, melhora do equilíbrio, da flexibilidade e da força de preensão manual em idosos.

\section{Palavras-chave}

Qualidade de Vida. Idosos. Equilíbrio. Força. 


\begin{abstract}
In recent times, there has been an increase in the elderly population, in front of it and aware of the increase in chronic diseases and also of the sedentary harm, strategies are necessary to maintain the active elderly. Physical activity supervised by physical therapists aim to preserve the function promoting a healthier lifestyle and better quality of life. The objective of this study was to investigate the effects of a physical therapy program on quality of life, balance, flexibility and grip strength in a group of elderly. As assessment was first applied Whoqol-brev questionnaire after the test wells bank in the sequence up and go test and finalize the manual dynamometer. The participants were evaluated at baseline and after 9 months of the program, over 35 sessions, and these were conducted in groups once a week with a duration of fifty minutes. The results show statistically significant improvement in perceived health status, flexibility, balance and manual MSD grip strength. It was concluded that a long-term physical therapy exercise program is an effective way to maintain quality of life, improve balance, flexibility and grip strength.
\end{abstract}

\title{
Keywords
}

Quality of life. Elderly. Balance. Force.

\section{Introdução}

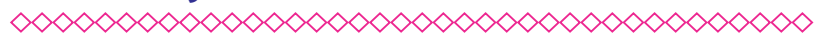

Nos últimos anos, observa-se um fenômeno mundial que ocorre devido a mudanças nos indicadores sociais de natalidade e mortalidade, evidenciando um aumento na expectativa de vida da população ${ }^{1}$. Isso ocorre tanto em países desenvolvidos como em desenvolvimento, como é o caso do Brasil. Segundo o Instituto Brasileiro de Geografia e Estatística (IBGE), no período entre 2001 a 2011, a população idosa aumentou de $9,1 \%$ para $12,1 \%^{2}$. Com o aumento da sobrevida da população, ressalta-se a importância de garantir aos idosos não apenas maior longevidade, mas felicidade, qualidade de vida e satisfação pessoal ${ }^{3}$.

0 envelhecimento pode ser definido como um processo dinâmico e progressivo, que gera alterações morfológicas, funcionais e bioquímicas ${ }^{4}$. 0 processo do envelhecimento associado ao estilo de vida moderno, em que a inatividade física, os hábitos alimentares inadequados e o estresse contribuem para o aumento das doenças crônicodegenerativas, maximizam as perdas funcionais comuns aos idosos ${ }^{5}$. Considera-se, assim, importante a criação de estratégias que colaborem com o engajamento de longo prazo do idoso em diferentes tipos de atividades físicas ${ }^{6}$.
Aatividade física regularéum meio de promoção de saúde e qualidade de vida, os programas mundiais de otimização a saúde destacam, por meio de evidencias epidemiológicas, o efeito positivo de uma vida ativa ou o envolvimento de um indivíduo em atividades físicas ${ }^{7}$.

Dentre as diversas funções do profissional fisioterapeuta, destaca-se a atuação na prevenção e promoção à saúde, visando a preservação das funções, adiamento da instalação de incapacidades e, de um modo geral, promovendo uma vida mais saudável e com melhor qualidade . $^{8}$

Diante disso, o objetivo deste estudo foi verificar os efeitos de um programa de fisioterapia na qualidade de vida, no equilíbrio, na flexibilidade e na força de preensão manual em um grupo de idosas da cidade de Erechim-RS.

\section{Materiais e métodos}

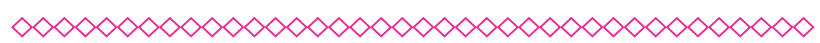

0 presente estudo caracteriza-se como um estudo longitudinal, não controlado, do tipo intervenção e com abordagem quantitativa. Realizado na cidade de Erechim, estado do Rio Grande do Sul, contemplou uma população de estudo composta por idosas residentes na mesma cidade. A amostra foi intencional, composta por 
15 idosas participantes do grupo de idosos do bairro Bela Vista. Foram incluídos na amostra participantes que concordaram com o Termo de Consentimento Livre e Esclarecido (TCLE), que tinham idade superior a 60 anos, sem a presença de doenças ortopédicas e reumáticas que limitassem a participação nas atividades, sem doenças agudas, com pressão arterial controlada, sem doenças cardíacas e pulmonares exacerbadas.

As participantes foram avaliadas antes e após nove meses da realização do programa de exercícios fisioterapêuticos, tal avaliação deuse a partir dos testes e questionários descritos a seguir. A qualidade de vida foi avaliada com 0 questionário WHOQOL-brev, esse questionário consta de 26 questões, sendo duas questões gerais de qualidade de vida e as demais (24) representam quatro domínios: físico, psicológico, relações sociais e meio ambiente ${ }^{\text {. }}$ O equilíbrio foi avaliado utilizando o teste "up and go" que avalia, também, as transferências de posição de sentado para em pé, estabilidade na deambulação e mudanças de curso da marcha, além do risco de quedas. Elaborado em 1991 por Podsiadlo e Richardson, o teste consiste em levantar-se de uma cadeira, caminhar três metros em linha reta, virar-se, retornar ao local de partida e sentar-se novamente ${ }^{10}$.

Para mensurar a flexibilidade foi realizado o banco de Wells que é utilizado para medir a flexibilidade da parte posterior do tronco e MMII. Para o teste, foram realizadas três repetições ${ }^{11}$, sendo que para a análise foi utilizada a melhor delas. Para avaliar a força de preensão manual foi utilizado um dinamômetro manual (Gamar®), foram realizadas três medições, com a utilização da melhor das repetições na análise. Os participantes foram instruídos a fazer uma contração máxima por três segundos em cada teste, havendo um período de descanso de 30 segundos entre cada teste ${ }^{12}$.

Após a avaliação inicial deu-se início ao programa de exercícios fisioterapêuticos realizados na Clinica Escola de Fisioterapia da Universidade Regional Integrada do Alto Uruguai e das Missões URI - Campus Erechim, sendo realizado uma vez por semana, com duração de cinquenta minutos, durante nove meses, sendo todas as 25 sessões acompanhadas de musicoterapia. O programa de exercícios foi composto por cinco etapas:

Etapa 1 - Aquecimento (danças e dinâmicas) realizado por cinco minutos.

Etapa 2 - Alongamentos ativos (MMSS: flexores, extensores, abdutores adutores, rotadores internos e externos de ombro, flexores e extensores de cotovelo e punho. MMII: flexores, extensores, abdutores e adutores de quadril, flexores e extensores de joelho dorsiflexores e plantiflexores. Coluna: flexores e extensores, rotadores e inclinadores de coluna lombar e cervical) com manutenção de 30 segundos em cada alongamento, totalizando 15 minutos.

Etapa 3 - Fortalecimento, realizado por 15 minutos e utilizando fita elástica, halter, caneleira, peso corporal, bastão, bola, entre outros, para as seguintes musculaturas: MMSS: flexores, extensores, abdutores adutores, rotadores internos e externos de ombro, flexores e extensores de cotovelo e punho. MMII: flexores, extensores, abdutores e adutores de quadril, flexores e extensores de joelho, dorsiflexores e plantiflexores.

Etapa 4 - Exercícios posturais usando peso corporal, bastão, bola, entre outros objetos para coluna cervical, torácica e lombar com ênfase para musculatura paravertebral e abdominal, realizados por dez minutos.

Etapa 5 - Relaxamento associado à respiração por cinco minutos.

Foram seguidas as diretrizes da Resolução $n^{\circ}$ 466 de 12 de dezembro de 2012, que aprova as normas e diretrizes regulamentadoras de pesquisa envolvendo seres humanos, do Conselho Nacional de Saúde do Ministério da Saúde. Para a análise dos dados foram utilizadas a estatística descritiva e o teste t de Student para amostras pareadas com o programa GraphPadPrism 6, considerando valores de $p<0,05$ significância estatística.

\section{Resultados e Discussão}

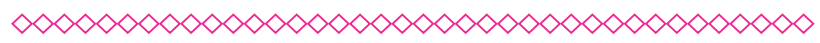

Esse estudo contou com a participação de 15 idosas do sexo feminino, com média de idade de 
70,4 $\pm 7,4$ anos, não praticantes de atividade física regula. Os resultados obtidos para a QDV antes e após a realização do protocolo de exercícios fisioterapêuticos podem ser observados na Tabela 1. Ao analisar os resultados observa-se uma melhora na percepção de qualidade de vida e na satisfação com a saúde, porém significância estatística apenas para a satisfação com a saúde $(p=0,041)$.

Nas Figuras 1 e $\mathbf{2}$ podem ser observados os resultados referentes à flexibilidade e ao equilíbrio. Nesse sentido, observa-se uma melhora estatisticamente significativa da flexibilidade $(p=0,002)$ antes e após o programa de fisioterapia, com valores que ultrapassaram $52 \mathrm{~cm}$ de flexibilidade.

A Figura 2 traz os resultados referentes ao equilíbrio, sobre o qual nota-se uma melhora em mais de dois segundos na capacidade de manter o equilíbrio dinâmico, mensurado com o teste up and go, que também demonstrou melhora estatisticamente significativa $(p=0,0001)$.

Na Figura 3 pode-se observar os resultados referentes à força de preensão manual, em que nota-se uma melhora, porém com significância estatística apenas para o membro superior direito, visto que o p apresentou-se igual a 0,0001, não apontando resultados estatisticamente significativos para o membro superior esquerdo, com $p=0,26$.

\section{Discussão}

$\infty \times \infty \times \infty \times \infty \times \infty \times \infty \times \infty \times \infty \times \infty \times \infty \times \infty \times \infty \times \infty \infty$

Diversos autores observaram a influência da atividade física na qualidade de vida de idosos e relatam que idosos praticantes de atividade física têm melhor qualidade de vida quando comparados aos sedentários ${ }^{13}{ }^{14}$. Outro estudo observou a QDV de idosas após a prática de três atividades distintas (dança, musculação e meditação), comparando os grupos de atividades com um grupo controle e evidenciando que os grupos dança e musculação alcançam melhores resultados na QDV quando comparados aos grupos meditação e ao grupo controle ${ }^{15}$
Autores demonstram a importância de intervenções fisioterapêuticas para a qualidade de vida. Ferreira ${ }^{16}$, por exemplo, observou a qualidade de vida de idosas antes e após uma intervenção fisioterapêutica, percebendo melhora em todos os quesitos analisados e destacando a importância da presença do fisioterapeuta no atendimento a grupos de idosos com foco em prevenir o aparecimento de doenças, retardar a senescência e melhorar a QDV dessa população.

A melhora da flexibilidade de idosos após a prática de exercícios físicos, como a observada no nosso estudo, já está bem documentada na literatura, ediversos autoresconfirmama melhora dessa variável após prática regular de exercícios físicos ${ }^{17,18}$. Outros autores demonstram melhora nas capacidades funcionais, como equilíbrio, agilidade, coordenação, força, flexibilidade e resistência ${ }^{19}$.

Dalla Corte e colaboradores ${ }^{20}$ ao analisarem a relação do equilíbrio com a flexibilidade de idosas praticantes e não praticantes de atividade física observam que os participantes de atividades físicas tiveram melhores índices das variáveis analisadas.

Em outro estudo, que analisou 30 idosos sedentários e ativos, observou-se que os idosos ativos tiveram melhores resultados no que diz respeito ao equilíbrio e à velocidade de caminhada. Tal estudo concluiu que a prática de atividade física contribui para a prevenção de quedas e melhora a função e a qualidade de vida dessa população²1.

No estudo realizado por Almeida, Veras e Doimo ${ }^{22}$, que compararam o equilíbrio estático e dinâmico de idosas praticantes de ginástica e hidroginástica, foi observado que não houve superioridade entre as modalidades nos quesitos analisados, porém houve uma tendência de superioridade do grupo ginástica em parâmetros como agilidade, equilíbrio e flexibilidade.

No presente estudo observou-se uma melhora na força manual do lado dominante, o que também ocorre em um estudo que observou os efeitos de exercícios resistidos no equilíbrio, mobilidade funcional e na qualidade de vida de idosas, destacando a melhora significativa da 
força muscular das idosas, além de melhora no equilíbrio e qualidade de vida ${ }^{23}$.

Já na pesquisa de Rebelatto ${ }^{4}$, que analisou a influência de um programa de atividade física de longa duração sobre a força manual e a flexibilidade corporal de mulheres idosas, aponta para a manutenção dos resultados inicias nos quesitos analisados.

\section{Considerações finais}

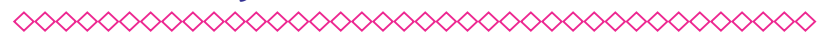

Conclui-se que um programa de exercícios fisioterapêuticos é um meio eficaz para a manutenção e melhora da qualidade de vida, melhora do equilíbrio, da flexibilidade e da força, além de prevenir quedas, o que, para essa população, significa a realização de movimentos mais refinados, coordenados e, dessa forma, contribuem para autonomia do idoso.

\section{Referências}

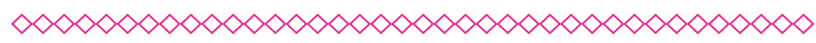

1. Carvalho MP, Luckow ELT, Peres W, Garcias GL, Siqueira FCV. O envelhecimento e seus fatores de risco associados. Rev. Bras. de Ciên. do Envelh. Hum. 2011;8(2):265-271.

2. Instituto Brasileiro De Geografia e Estatísticas (IBGE): Censo Demográfico 2012. http://www. Censo 2012.ibge.gov.br (acesso em: 06/06/2015).

3. Joia LC, Ruiz T. Donalisio MR. Condições Associadas ao Grau de Satisfação com a Vida entre a População de Idosos. Rev. Saúde. Públ. 2007;41(01):131-138.

4. Rebelatto JR, Calvo J, Orejuela JR, Portillo JC. Influência de um Programa de Atividade Física de Longa Duração Sobre a Força Muscular Manual e a Flexibilidade Corporal de Mulheres Idosas. Rev. bras. Fisioter. 2006;10(1):127-132.

5. Tribess S. Percepção da imagem corporal e fatores relacionados à saúde em idosas. [Dissertação de Mestrado] Florianópolis SC Universidade Federal de Santa Catarina, 2006.
6. Gouveia JAG, Volpato IA, Gilberto PF, Benedeti MR, Bertolini SMG, Oliveira AP. Equilíbrio em praticantes de dança sênior em um centro dia do idoso. Rev. Cien. JOPEF. 2013;15(2):24-31.

7. Mazo GZ, Cardoso FL, Aguiar DL. Programa de Hidroginástica para Idosos: Motivação, Autoestima e Auto-imagem. Rev. bras. cineantropom. desempenho hum. 2006;08(02):67-72.

8. Guimarães LHCT, Galdino DCA, Martins FLM, Abreu SR, Lima M, Vitorino DFM. Avaliação da Capacidade Funcional de Idosos em Tratamento Fisioterapêutico. Rev. Neurociênc. 2004;12(3):130-133.

9. Fleck MPA, Louzada S, Xavier M. Chachamovich E. Vieira G, Santos L. Pinzon V. Aplicação da versão em português do instrumento abreviado de avaliação da qualidade de vida "WHOQOL-bref". Rev. Saúde.Públ. 2000;34(2):178-183.

10. Podsiadlo D. Richardson S. The Timed "Up and Go". A test of Basic Functional Mobility for Frail Elderly Persons. J. Am. Geriatr. Soc. 1991;39(2):06-12.

11. Wells KF, Dillon EK. The sit and reach: a test of back and leg flexibility. Res. Q. Exercise Sport. 1952;23(05):115-118.

12. Reis MM, Arantes PMM. Medida da força de preensão manual: validade e confiabilidade do dinamômetro SAEHAN. Fisioter. Pesqui. 2011;18(2):176-181.

13. Pimenta FAP, Simil FF, Tôrres HOG, Amaral CFS, Rezende CF, Coelho TO, Rezende NA. Avaliação da qualidade de vida de aposentados com a utilização do questionário SF-36. Rev. Assoc. Med. Bras. 2008;54(1):55-60.

14. Toscano JJO, De Oliveira ACC. Qualidade de Vida em Idosos com Distintos Níveis de Atividade Física. Rev. Bras. Med. Esporte 2009;15(3):169173.

15. Castro JC, Bastos FAC, Cruz THP, Giani TS, Ferreira MA, Dantas HM. Níveis de qualidade de vida em idosas ativas praticantes de dança, musculação e meditação. Rev. Bras. Geriatr. Gerontol 2009;12(2):255-265. 
16. Ferreira LL. Análise da Qualidade de vida de Idosos antes e após intervenção Fisioterapêutica. Rev. Inspirar 2010;2(5)18-21.

17. Beserra LRS. Análise da flexibilidade em idosos participantes de atividades físicas no SCFV Serraria. [Trabalho de Conclusão de Curso] Borborema DF Universidade de Brasília - Pólo Duas Estradas, 2014.

18. Tonon PP, Duim EL, Santos SS. Efetividade da fisioterapia associada à dançaterapia na melhora da capacidade aeróbia e flexibilidade de mulheres idosas com histórico de quedas. Rev. Saúde Pesqui. 2012;5(3):517-524.

19. Cipriani NCS, Meurer ST, Benedetti TRB, Lope MA. Aptidão funcional de idosas praticantes de atividades físicas. Rev. Bras. Cineantropom. Desempenho Hum. 2010;12(2):106-111.

20. Dalla Corte FMA, Vieira LM, Moreira P, Gessinger CF Noll M. Relação do equilíbrio com a flexibilidade de idosos praticantes e não praticantes de exercícios físicos. Cinergis. 2012;13(3):27-35.

21. Silva TO, Freitas RS, Monteiro MR, Borges SM. Avaliação da capacidade física e quedas em idosos ativos e sedentários da comunidade. Rev. Bras. Clin. Med. 2010;8(5):392-398.

22. Almeida APPV, Veras RP, Doimo LA. Avaliação do equilíbrio estático e dinâmico de idosas praticantes de hidroginástica e ginástica. Rev. Bras. Cineantropom. Desempenho Hum. 2010;12(1)55-61.

23. Prado FA, Egydio PRM, Teixeira ALC, Izzo P. A influência dos exercícios resistidos no equilíbrio, mobilidade funcional e na qualidade de vida de idosas. O Mundo da Saúde. 2010;34(2)183-191. 


\section{Anexos}

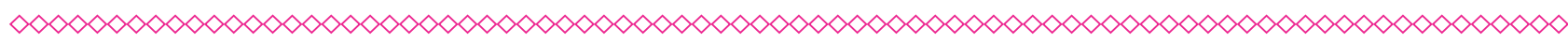

Tabela 1 - Dados obtidos a partir da aplicação do questionário Whoqol- Brev, antes e após a realização do programa de exercícios fisioterapêuticos, no grupo de idosas $(n=15)$. Dados apresentados por domínios de Qualidade de Vida.

\begin{tabular}{|l|c|c|c|}
\hline \multicolumn{1}{|c|}{ Domínios } & $\begin{array}{c}\text { Início } \\
(\mathbf{X})\end{array}$ & $\begin{array}{c}\text { Final } \\
(\mathbf{X})\end{array}$ & Valor de $\mathrm{P}$ \\
\hline Pecepção Qualidade de vida & 4 & 4,06 & $\mathrm{p}=0,670$ \\
\hline Satisfação com saúde & 3,8 & 4,06 & $\mathrm{p}<0,041$ \\
\hline Domínio Físico & 3,9 & 3,78 & $\mathrm{p}=0,204$ \\
\hline Domínio Psicológico & 4,01 & 3,95 & $\mathrm{p}=0,489$ \\
\hline Domínio Relações Sociais & 4,28 & 4,18 & $\mathrm{p}=0,357$ \\
\hline Domínio Meio Ambiente & 4,88 & 3,94 & $\mathrm{p}=0,0651$ \\
\hline
\end{tabular}

(clique para voltar ao texto)

Figura 1 - Dados da flexibilidade obtidos com a utilização do banco de Wells no grupo de idosas, antes e após a realização do programa de exercícios fisioterapêuticos.

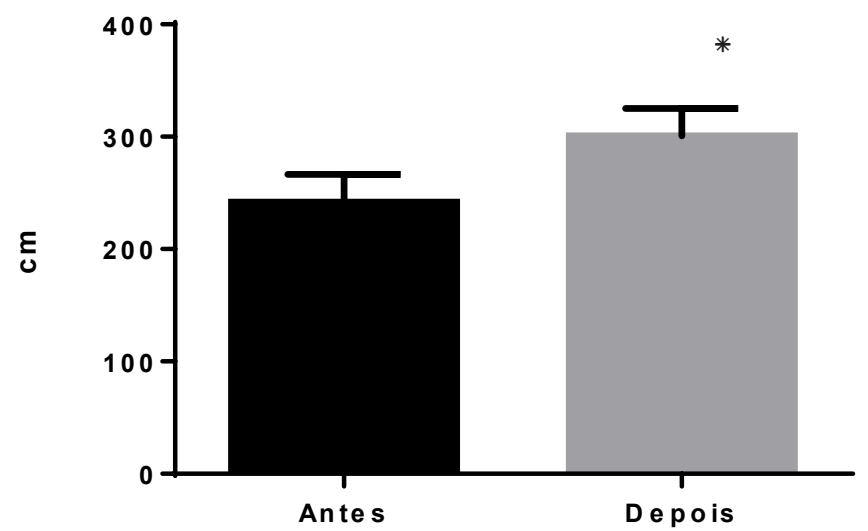

* Resultados estatisticamente significativos $(\mathrm{p}<0,05)$. Teste t de Student para amostras pareadas. 
Figura 2 - Dados referentes ao equilíbrio obtidos com a realização do teste up and go no grupo de idosas, antes e após a realização do programa de exercícios fisioterapêuticos.

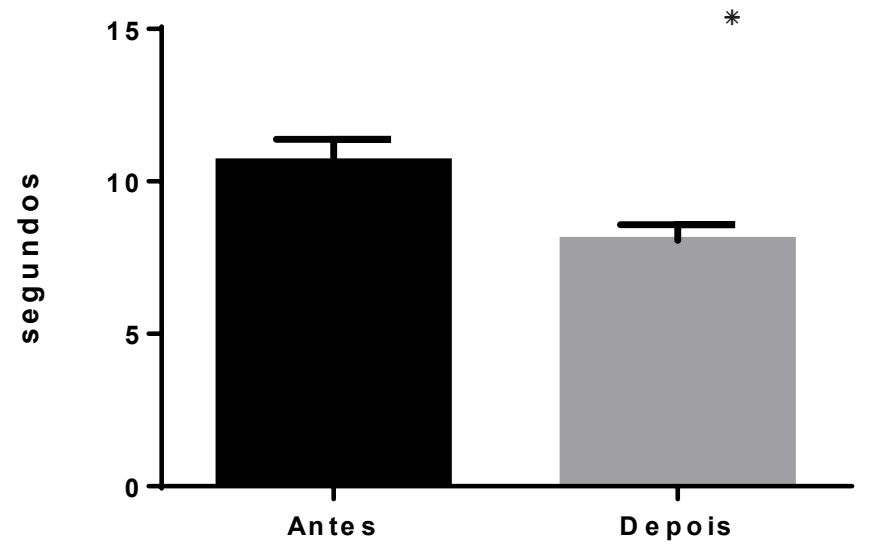

* Resultados estatisticamente significativos $(\mathrm{p}<0,05)$. Teste t de Student para amostras pareadas.

(clique para voltar ao texto)

Figura 3 - Dados referentes à força de preensão manual do membro superior direito e esquerdo obtidos por meio da dinamometria manual no grupo de idosas, antes e após a realização do programa de exercícios fisioterapêuticos.

MSD

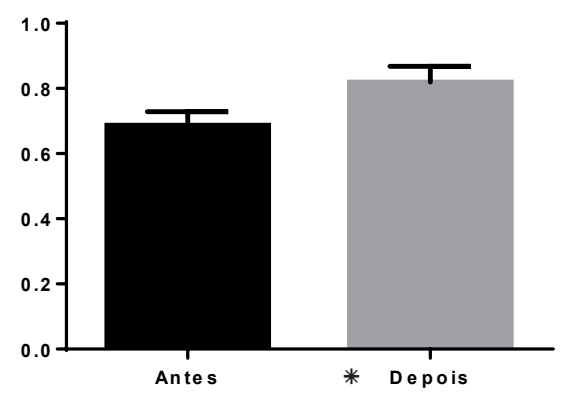

MSE

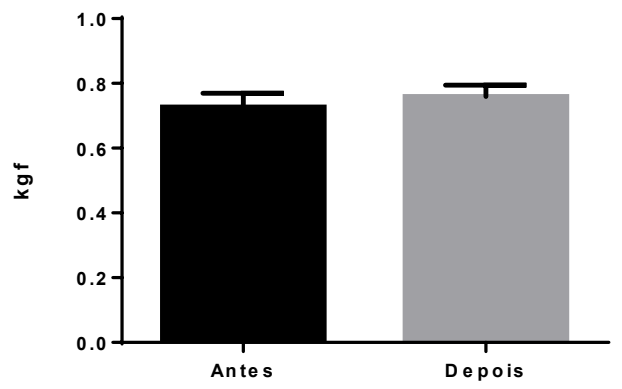

* Resultados estatisticamente significativos $(p<0,05)$. Teste t de Student para amostras pareadas. 